
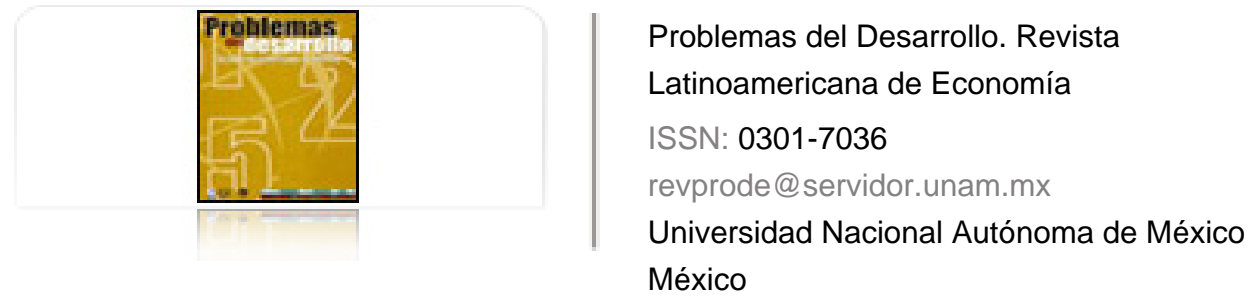

Musacchio, Andrés

LAS OSCILACIONES CÍCLICAS DE LA ECONOMÍA ARGENTINA EN LAS DOS ÚLTIMAS

DÉCADAS

Problemas del Desarrollo. Revista Latinoamericana de Economía, vol. 40, núm. 159, octubrediciembre, 2009, pp. 143-168

Universidad Nacional Autónoma de México

Distrito Federal, México

Disponible en: http://www.redalyc.org/articulo.oa?id=11820103008

Cómo citar el artículo

Número completo

- Más información del artículo

Página de la revista en redalyc.org

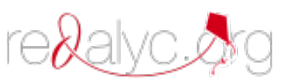

Sistema de Información Científica

Red de Revistas Científicas de América Latina, el Caribe, España y Portugal Proyecto académico sin fines de lucro, desarrollado bajo la iniciativa de acceso abierto 


\title{
LAS OSCILACIONES CÍCLICAS DE LA ECONOMÍA ARGENTINA EN LAS DOS ÚLTIMAS DÉCADAS ${ }^{1}$
}

\author{
Andrés Musacchio*
}

Fecha de recepción: 18 de junio de 2009. Fecha de aceptación: 8 de septiembre de 2009.

\section{Resumen}

El artículo se propone analizar de manera conjunta las brechas externa y fiscal, tratando de evaluar la consistencia macroeconómica de las políticas argentinas en las últimas dos décadas. La cuestión reviste gran importancia, pues para un país como Argentina, con una alta deuda externa pública, existe una necesidad estructural de lograr superávit externos y fiscales que generen los recursos para el pago del servicio de dicha deuda.

Así, se llega a la conclusión de que en tiempos del Plan de Convertibilidad, las etapas expansivas generaban un déficit externo, mientras en las etapas recesivas aparecía un déficit fiscal. De una u otra forma, la política se sostenía a costa de un endeudamiento creciente.

Palabras clave: Argentina, brecha externa, brecha fiscal, consistencia macroeconómica, endeudamiento externo.

* Investigador del Instituto de Estudios Históricos, Económicos Sociales e Internacionales, Facultad de Ciencias Económicas de la Universidad de Buenos Aires. Correo electrónico: andresmusacchio@hotmail.com

1 Este artículo forma parte del proyecto UBACYT E-808. El autor agradece a Verónica Robert, Pablo Lavarello y Miguel Zanabria por las discusiones en torno a una versión anterior del texto. También a Noemí Brenta por sus precisos comentarios. Los problemas y errores del texto son, como es habitual, responsabilidad exclusiva del autor. 
Abstract

The article proposes analyzing, in an integrated way, the external and fiscal gaps, trying to evaluate the macroeconomic consistency of Argentina's policies in the last two decades. The question is of great importance, because for a country like Argentina, with a high external public debt, a structural need exists to achieve external and fiscal surpluses that generate resources to pay for the servicing of this debt.

It therefore comes concludes that at the time of the Convertibility Plan, the expansionary stages generated an external deficit, while during the recessionary stages a fiscal deficit appeared. In one way or another, the policy was sustained at the cost of a growing indebtedness.

Key words: Argentina, external gap, fiscal gap, macroeconomic consistency, external indebtedness.

\section{Résumé}

L'article a pour propos d'analyser de manière conjointe les brèches externe et fiscale, en essayant d'évaluer le bien fondé macroéconomique des politiques argentines durant les deux dernières décennies. La question est très importante car un pays comme l'Argentine, ayant une dette publique externe élevée, est sujet à un besoin structurel d'obtenir des excédents externes et fiscaux qui engendrent les ressources pour le paiement du service de la dite dette.

On aboutit ainsi à la conclusion qu'à l'époque du Plan de Convertabilité, les étapes expansives généraient un déficit externe, alors que pendant les étapes récessives aparaissait un déficit fiscal. D'une manière ou d'une autre, la politique était maintenue au prix d'un endettement croissant.

Mots clés: Argentine, brèche externe, brèche fiscale, bien fondé macroéconomique, endettement externe.

\section{Resumo}

$O$ artigo se propõe analisar de maneira conjunta as brechas externa e fiscal, tratando de avaliar a consistência macroeconômica das políticas argentinas nas últimas duas décadas. A questão tem grande importância, pois para um país como a Argentina, com uma alta divida externa pública, existe uma necessidade estrutural de lograr superávits externos e fiscais que gerem os recursos para o pagamento do serviço de tal divida.

Assim, chega-se à conclusão de que nos tempos do Plano de Convertibilidade, as etapas expansivas geravam um déficit externo, enquanto que nas etapas recessivas aparecia um déficit fiscal. De uma forma ou de outra, a política se mantinha às custas de um endividamento crescente.

Palavras-chave: Argentina, brecha externa, brecha fiscal, consistência macroeconômica, endividamento externo. 


\section{Introducción}

\section{De la crisis interna a la crisis internacional}

a crisis de 2001 es la peor de cuantas han asolado al país en toda su historia.
A diferencia de otros estallidos, se combinaron crisis en el orden político,
social y económico. En este último caso, no sólo se trató de una crisis financiera, sino también abarcó la producción, las características de la inserción internacional y las pautas normativas. Por eso se transformó en una bisagra que separa dos etapas y permite, por un lado, ver los problemas del neoliberalismo y, por el otro, observar una recuperación -impulsada mediante políticas heterodoxas- tan sorprendente como la crisis precedente.

Una crisis tan amplia no puede responder a una causa única. Tal vez esa complejidad ha transformado su análisis en un desafío plasmado en numerosos trabajos y debates. En ellos, algunas posiciones intentan reducir la interpretación a una o dos causales, mientras otros prefieren reconstruir una trama más matizada, aunque privilegien algunos argumentos ${ }^{2}$. Así, hay autores que enfatizan factores externos y, especialmente, los cambios en la liquidez de los mercados financieros ${ }^{3}$; pero la mayoría acentúa el peso de los factores internos, pues las transformaciones externas fueron iguales para todo el mundo y, salvo Turquía, ningún país tuvo un colapso tan severo.

El reconocimiento de lo interno como principal factor explicativo no significa un acuerdo respecto de los disparadores y los encadenamientos. Para la visión ortodoxa, la crisis se ciñó al abandono del régimen de convertibilidad sin la adopción de una regla monetaria aún más severa, como la dolarización. El análisis pone énfasis en la irresponsabilidad fiscal, al no bajar el gasto en el auge, generando complicaciones cuando el mercado financiero global se volvió reticente a prestar a los países emergentes. Como complemento, se critica el no haber privatizado oportunamente servicios públicos como salud y educación, así como la falta de flexibilidad en el mercado de trabajo ${ }^{4}$.

En las interpretaciones heterodoxas, los problemas estructurales tienen un despliegue mucho más largo ${ }^{5}$, ya sea por la conformación de las estructuras que engendraron la crisis o por los ciclos que se repiten desde entonces, donde la última crisis

Para un análisis crítico de las diferentes posturas, cfr. Mario Rapoport et al., 2006.

Cfr. Calvo e Izquierdo, 2002.

Cfr. Rodríguez, 2000; Mussa, 2002; Artana, López Murphy y Navajas, 2004.

Muchos entienden, incluso, que la matriz del análisis debe incluir todo el periodo que se inicia con la dictadura de 1976. 
sería la más grave pero no la única ${ }^{6}$. La crisis combina aspectos financieros con un debilitamiento productivo, y afecta al empleo, la distribución del ingreso y al proceso de acumulación. Los análisis heterodoxos cuestionan las políticas públicas de manera opuesta al planteamiento ortodoxo; la combinación de apertura comercial, liberalización financiera y retraso cambiario se habría sumado a la ausencia de políticas activas para promover la producción y el empleo y a una intervención insuficiente en la regulación de los mercados ${ }^{7}$. En este marco, se destacan aspectos específicos como la crisis fiscal, el excesivo endeudamiento, las inconsistencias de la estrategia de inserción internacional o las dificultades para mantener un régimen cambiario como aspectos de un proceso complejo, que anuda la trama económica con los procesos sociales e históricos ${ }^{8}$. Algunos trabajos enfatizan el sostenimiento de las políticas que llevaron a la crisis como resultado del comportamiento de los grupos económicos locales e internacionales y de las características del proceso de acumulación y fuga de capitales9. También la dimensión que articula las políticas y los intereses internos con grupos económicos, políticos y financieros externos, así como con los organismos financieros internacionales, es una matriz que debe ser analizada con detenimiento al desentrañar esta parte de la trama ${ }^{10}$.

Un aspecto de la crisis surge, sin embargo, de una inconsistencia técnica que tornaba incompatibles en el largo plazo los equilibrios externo y fiscal, problema que abordamos en el presente artículo. Esa inconsistencia podría anticipar el advenimiento de la crisis desde el inicio mismo del Plan de Convertibilidad. Asimismo, generaba una necesidad de financiar el déficit correspondiente a deuda pública, en el caso fiscal, y a deuda externa, en el caso de la cuenta corriente. Por lo tanto, la incompatibilidad solamente podía mantenerse a costa de un ingreso permanente de capitales, que tornaba al modelo económico puesto en marcha absolutamente dependiente de la coyuntura externa.

En un país con un fuerte endeudamiento externo público, una política "sustentable" debe lograr articular en el largo plazo un superávit en la balanza comercial con excedente en el presupuesto. Se intentará mostrar que las políticas aplicadas

Cfr. Rapoport et al., 2006; Becker, 2003; o Vitelli, 2002.

Éstos son, por ejemplo, los lineamientos presentes en las principales aportaciones al Plan Fénix (2002; 2003).

8 En Boyer y Neffa (2004), se compilan diversos trabajos que reflejan los puntos comunes y las diferencias de las diversas vertientes heterodoxas.

9 Cfr. Basualdo, 2002; 2003.

10 Cfr. Rapoport et al., 2006; o Minsburg, 2003. 
impidieron tal "principio de coherencia" y que, en función del momento del ciclo, el superávit en una brecha invariablemente se conjugaba con déficit en la otra. Éstas son las ideas que presentaremos en la primera parte de este artículo, con un modelo analítico que nos permita visualizar gráficamente las hipótesis expuestas. No buscamos entender las "causas en última instancia" de la crisis ni explicar por qué, a pesar de las inconsistencias, las políticas se mantuvieron inamovibles. No obstante, el problema aquí analizado no carece de relevancia pues no sólo ayuda a comprender el desarrollo de la crisis, sino permite analizar la consistencia de las políticas económicas actuales, pues la restricción externa continúa vigente. Por lo tanto, de la habilidad para alterar los parámetros fiscales y comerciales depende que la crisis no vuelva a repetirse.

La conjunción de brechas externa y fiscal discordantes fue revertida a partir de 2003. Desde entonces, se produjo un crecimiento acelerado con superávit comercial y fiscal, que permitió cancelar gran parte de los servicios de la deuda reprogramados, acumular reservas y mostrar un cuadro excepcionalmente sólido en las finanzas públicas. ¿Fue ello el fruto de una transformación estructural o el resultado de una coyuntura especial y de un par de medidas ingeniosas pero sin sustento en el largo plazo? Los cambios y las razones que le dieron a la expansión de la década de 2000 una sustentabilidad ausente en la de 1990 y que evitaron tensiones explosivas en el mercado financiero, en el endeudamiento o en las cuentas públicas, serán el eje de la segunda parte de este trabajo.

La coyuntura actual reaviva la discusión, pues la crisis internacional alteró las condiciones de liquidez financiera que había enmarcado la política interna desde 2003. El tema de la deuda externa, que parecía superado luego de la renegociación con los acreedores privados y de la cancelación de los pasivos con el FMI, resurge con la súbita falta de liquidez internacional. De la misma forma, el derrumbe de la demanda externa y la caída de los precios en las exportaciones afectan la sólida posición de divisas que venía acumulándose en las arcas públicas. Esto hace suponer que la espectacular recuperación esconde también algunas fragilidades, que remiten a problemas estructurales no resueltos y que pueden frustrar la continuidad del camino ascendente. Otra vez, el análisis de las brechas externa y fiscal da algunas pistas respecto de la manera en que la crisis penetra en Argentina; asimismo, muestra la necesidad de impulsar una activa política de desarrollo si se pretende evitar que aquélla corroa las bases de la recuperación. 


\section{Un modelo de brechas externa y fiscal como formas de aproximación a la crisis}

Un factor que permite entender la crisis de 2001 es la inconsistencia de la política económica aplicada desde 1991. Esa situación refleja una inconsistencia aún más profunda: la forma que asume la relación entre las estructuras financieras y productivas. En una economía con una elevada deuda externa pública, como la argentina posterior a la dictadura militar de 1976-1983, hay dos condiciones de consistencia para las políticas económicas. En primer término, el pago de la deuda en moneda fuerte supone la necesidad de lograr un superávit comercial que provea divisas genuinas para las transferencias. En segundo término, la adquisición de las divisas por parte del sector público supone la obtención de un superávit fiscal primario equivalente, a menos que se recurra a una masiva emisión de dinero o de títulos públicos internos. Estas dos restricciones pueden eludirse temporalmente con nuevas deudas o con inversiones directas, o postergando pagos; pero, a largo plazo, la restricción es inflexible.

Tanto la restricción externa como la fiscal se enlazan por medio de la relación que cada una tiene con la evolución del producto. Desde esa perspectiva, una política consistente en el largo plazo busca que los parámetros que vinculan al sector externo y a las cuentas públicas con el ingreso sean compatibles con las necesidades macroeconómicas. Analicemos estos aspectos en detalle.

El saldo comercial de un país se encuentra determinado, obviamente, por la magnitud de las exportaciones y la de las importaciones. Mientras en el mediano plazo resulta muy difícil influir sobre el nivel de exportaciones, ya que éstas tienen que ver con factores estructurales sobre los que se tiene poco control, como la demanda externa, la especialización comercial o el nivel de precios, las compras externas guardan una estrecha relación con el nivel interno de actividad ${ }^{11}$. Para un país altamente endeudado, y sin inversiones en el exterior que produzcan utilidades, el único ingreso "genuino" de divisas se origina en un saldo comercial positivo. La atracción de fondos desde el exterior, como muestra la experiencia de las tres últimas décadas, requiere condiciones especiales que garanticen ganancias extraordinarias, a costa de ruinosas consecuencias sobre el aparato productivo.

Las fluctuaciones del producto tienen una influencia importante sobre la balanza comercial, ya que su crecimiento suele causar incrementos en la demanda de productos importados, ya sea destinados al consumo o al propio proceso productivo, como

11 Lo anterior no implica de ninguna manera sostener la imposibilidad de alterar el volumen de exportaciones en el largo plazo por medio de políticas de promoción de las ventas externas o de fortalecimiento de la producción interna. 
insumos o bienes de capital. En cambio, las exportaciones no son impulsadas por el crecimiento, y por lo tanto su variación es independiente de la variación del producto. Por eso puede asumirse una relación inversa entre las variaciones de la producción y el saldo comercial: cuando la economía crece se impulsan las importaciones y se reduce el saldo comercial. Formalmente, podemos decir que

$$
S B C=F(P B I) ; F^{\prime}<0 \text {; donde } S B C \text { es el saldo de la balanza comercial. }
$$

Gráficamente, la relación adquiere la forma de la curva SC (véase ilustración 1). La curva mencionada, de pendiente negativa, muestra la evolución del saldo comercial a medida que se expande el PIB. La pendiente de la curva está influida por distintos parámetros, como la sensibilidad de las importaciones a la variación del producto (mucho mayor cuanto menos diversificada es la economía), la competitividad de las exportaciones, el tipo de cambio, las restricciones al ingreso de productos importados o del ingreso de productos propios a los mercados externos, etcétera. Así, aunque la relación entre ambas variables sea negativa, la política económica tiene márgenes de maniobra para modificar parcialmente el vínculo, alterando la pendiente de la curva o incluso desplazándola, por ejemplo, con una política de sustitución de importaciones o de menor diversificación productiva.

Por otra parte, las cuentas fiscales generalmente se comportan en el mismo sentido que el ingreso. Un aumento del producto no supone un aumento significativo del gasto (aunque sí pueda observarse lo contrario), pero impacta positivamente sobre los ingresos públicos. El mayor nivel de actividad genera un nivel creciente de ingresos impositivos y de cargas sociales. Por el contrario, cuando el producto se contrae, también lo hacen los ingresos públicos, deteriorando el equilibrio fiscal. Esto puede formalizarse como

$$
S F=G(P B I) ; G^{\prime}>0 ; \text { donde } \mathrm{SF} \text { es el saldo fiscal. }
$$

Gráficamente, la relación se visualiza en la curva SF de la ilustración 1. De su comportamiento se desprende que el superávit primario necesario para cubrir la segunda restricción apuntada -un superávit suficiente para afrontar el servicio de la deuda pública- sólo pueda alcanzarse con una expansión productiva sostenida.

Como señalamos, una política económica sustentable para un país que tiene un alto grado de endeudamiento, debe combinar un superávit comercial y fiscal al menos equivalentes al servicio de su deuda, como condición para poder sostenerse en 
Ilustración 1

Saldos comercial y fiscal y condición de coherencia

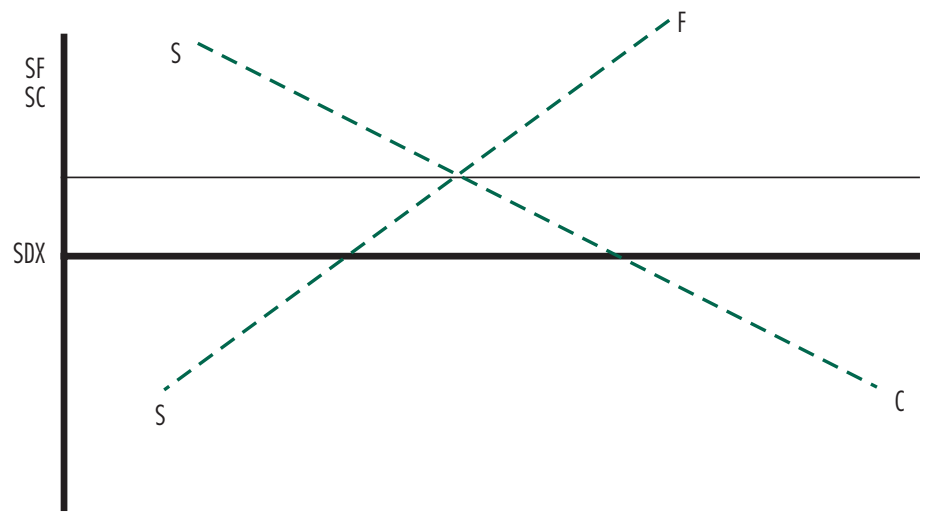

el tiempo sin generar un incremento paulatino y explosivo del endeudamiento. Esa condición de coherencia, es decir, el monto del servicio de la deuda, es la recta SDX de la ilustración.

\section{La inconsistencia entre las dos brechas en la década de 1990 como causa de la crisis}

Precisamente en esta cuestión puede encontrarse una inconsistencia mayor en la política económica que llevó a la crisis de 2001, pues el resultado de la política económica fue que las brechas externa y fiscal resultaran incompatibles. Por una parte, el plan afectó negativamente diversos aspectos de la relación entre producto y saldo comercial, provocando déficit a niveles menores del PIB que en la década de 1980. Enseguida, se enlistan las principales causas:

- Con el retraso cambiario, las exportaciones perdieron competitividad, recuperada sólo parcialmente con incrementos en la productividad.

- El retraso cambiario y la apertura abarataron artificialmente las importaciones. Así, muchas empresas reemplazaron bienes de capital e insumos nacionales por importados, y una parte importante del consumo se desvió hacia las importaciones.

- El incremento -inducido por la propia política económica- del precio relativo de los servicios luego de las privatizaciones también restó competitividad a las exportaciones y a la producción local frente a los bienes importados.

- La política de tipo de cambio fijo -llave del programa-, que mantuvo durante su primer tramo una tasa de inflación positiva relativamente alta, generó las condiciones para promover las importaciones y desalentar las exportaciones. 
- El paulatino aumento del costo financiero, especialmente la diferencia creciente entre la tasa de interés interna y la internacional, operó en el mismo sentido antes apuntado.

- La gran volatilidad de los precios internacionales de las exportaciones le confiere a la relación misma un grado importante de volatilidad. En la década de 1990, luego de una recuperación al comienzo, los términos del intercambio se estabilizaron, volvieron a mejorar en 1996 y, a partir de entonces, comenzaron una curva descendente hasta 2000.

Por esas razones, la política económica fue determinando un nivel mayor de importaciones por unidad de producción o, lo que es lo mismo, que el equilibrio comercial se alcanzara a niveles menores de producto que antes. Eso significó que los periodos expansivos generaran importantes déficit comerciales, como 1992-1994 o 1996-1997, mientras que el equilibrio se aproximaba en momentos de recesión, como en 1995 o a finales de esa década. La restricción de largo plazo tornaba incompatible el equilibrio externo con un proceso de crecimiento sostenido.

En el recuadro izquierdo de la gráfica 1, puede verse que tanto el comportamiento inverso de las tendencias del PIB y del saldo comercial, como sus cambios a lo largo de la década muestran un comportamiento como el que acabamos de describir. Además, cabe señalar que el superávit comercial necesario para equilibrar el sector externo se incrementó sin cesar a lo largo del periodo: el incremento del endeudamiento y la elevación de las tasas de interés obligaban a desembolsos crecientes, de manera que mientras la restricción externa se tornaba mas rígida, las necesidades de divisas aumentaban paulatinamente.

Gráfica 1

Evolución comparada del saldo comercial y la actividad economica (izq.) y línea SC (der.) 1993-2001
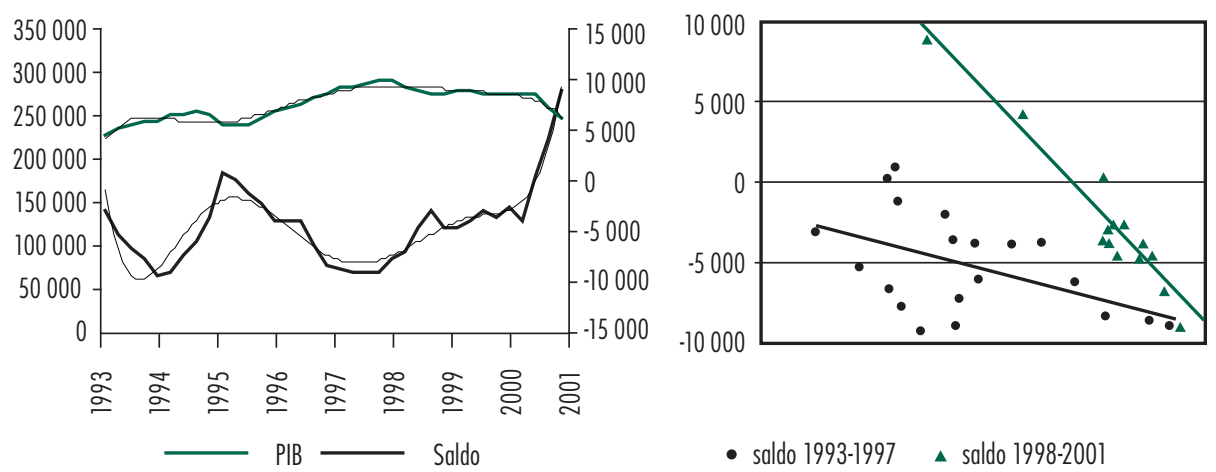

Fuente: Elaboración propia con base en información de INDEC. La línea SC fue construida con datos trimestrales desestacionalizados. 
Por otra parte, las inversiones directas pronto incidieron negativamente en la cuenta corriente por la transferencia de utilidades, mientras incrementaban más que proporcionalmente la demanda de importaciones. A su vez, los problemas señalados se agravaron continuamente. A lo anterior se sumó el paulatino efecto del Plan de Convertibilidad sobre la actividad productiva, que tornó cada vez más sensible la variación del saldo comercial en función de los cambios en el producto (véase recuadro derecho, gráfica 1). Esa mayor sensibilidad se aprecia en una línea SC mucho más vertical para el periodo 1998-2001 que la de 1993-1997.

Hay que considerar, además, el factor estructural de la función preponderante de las finanzas, estrechamente vinculadas a un contexto altamente especulativo abonado por un rasante crecimiento del endeudamiento externo, que intensificaba el patrón de especialización de las exportaciones en un conjunto reducido de commodities $^{12}$, es decir, la existencia de una base exportadora reducida a un puñado de actividades capaces de competir con las ganancias extraordinarias que ofrecía el sector financiero.

En cuanto a la brecha fiscal, es posible ver también la asociación, en este caso positiva, entre el saldo y el PIB, que permite explicar por qué en los años de crecimiento la ecuación fiscal encontró un nivel razonable, mientras que en las recesiones se convirtió en una fuente de problemas casi sin solución. Al igual que en el sector externo, la política económica afectó negativamente la relación entre cuentas públicas y nivel de ingreso. Desde un punto de vista estático, he aquí algunos de los factores que incidieron en ello:

- La privatización de los ingresos de la seguridad social desfinanció por completo el viejo sistema de jubilaciones y pensiones, que continuó a cargo del Estado. Por eso, debió recurrirse masivamente al crédito que otorgaron los propios fondos de pensión, con la paradoja de que el Estado continuaba recibiendo los fondos, pero ahora con un elevado costo financiero y con la obligación de devolverlos en el futuro, mientras que antes recibía transferencias sin costo financiero ni obligación de devolución.

- La reducción de aportaciones patronales para que de esa manera el sector privado recuperara la competitividad perdida por el retraso cambiario.

- La reforma impositiva que acentuaba la función de los impuestos indirectos, que dependen del nivel de ingresos y se vieron afectados por la redistribución regresiva del ingreso.

- El mantenimiento de fuertes subsidios a las empresas privatizadas, a pesar de que su transferencia debía suponer, para el Estado, un menor nivel de gasto.

12 Cfr. Musacchio, 2004b.

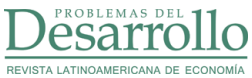


Desde el punto de vista dinámico, la elevación del endeudamiento y el incremento de las tasas de interés aumentaron paulatinamente el nivel del gasto por la incidencia de los servicios de la deuda, de modo que el equilibrio fiscal demandó, con el correr del tiempo, niveles crecientes de ingreso. Los ajustes introducidos en otras partidas de gastos (que, además, fortalecieron la tendencia al crecimiento de la participación del pago de la deuda en el gasto total), no hicieron sino agravar la situación, por su efecto recesivo que repercutía negativamente sobre los ingresos públicos.

Como puede verse en el recuadro izquierdo de la gráfica 2, al inicio de la década de 1990 se observa un deterioro del superávit en relación con una evolución más estable del PIB, que nos indica un paulatino desplazamiento de la curva SF de la derecha.

Gráfica 2

Evolución comparada del superávit primario

y la actividad económica (izq.) y curva SF (der.), 1994-2001

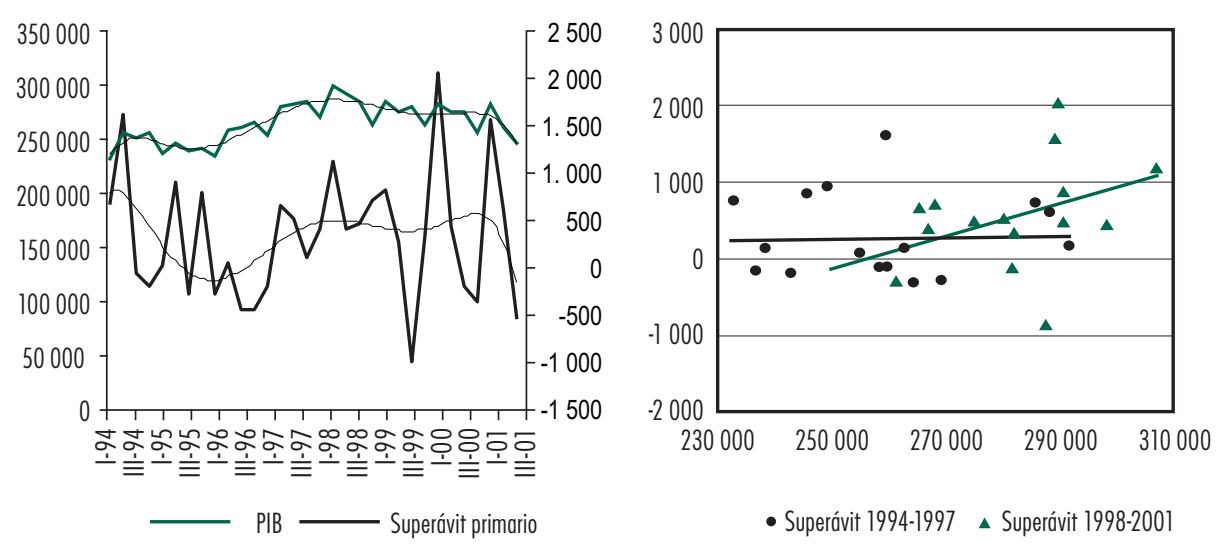

Fuente: Elaboración propia con base en información de INDEC.

El desplazamiento muestra una relación de baja sensibilidad en el primer tramo del plan, pero aumenta con el transcurso del tiempo, lo que gráficamente se observa en la mayor inclinación de la curva SF.

La evolución de ambas brechas permite observar que la política económica de la década de 1990 estuvo lejos de garantizar la coherencia requerida para una sustentabilidad del programa. Como puede verse en el cuadro 1, casi en ningún momento los saldos comerciales y fiscales alcanzaron a satisfacer los pagos de intereses. De esa forma, fueron acumulándose servicios impagos, cancelados con nuevas deudas, pero que, a la postre, alejaban aún más a la economía del "punto de coherencia". 
Bajo estas condiciones, pueden identificarse algunas características del modelo de convertibilidad que contribuyeron a darle cuerpo a la crisis con la que se derrumbó el modelo. Como acabamos de señalar, una de sus características intrínsecas fue el constante endeudamiento, clave para que el bache fiscal no se convirtiera en un inmediato factor de quiebre del programa. A diferencia de lo que se plantea en los estudios ortodoxos, el problema principal no era un Estado excesivamente dispendioso. El gasto corriente tendió a mantenerse en niveles razonables, y fue insuficiente para atender las necesidades de infraestructura o las crecientes demandas sociales que generaba la pésima distribución del ingreso ${ }^{13}$. En todo caso, el problema del gasto fue in crescendo con el galopante incremento de la deuda.

Cuadro 1

Principales variables observadas (millones de pesos / *dólares)

\begin{tabular}{|l|r|r|r|r|r|r|r|r|r|}
\hline & \multicolumn{1}{|c|}{1993} & \multicolumn{1}{c|}{1994} & \multicolumn{1}{c|}{1995} & \multicolumn{1}{c|}{1996} & \multicolumn{1}{c|}{1997} & \multicolumn{1}{c|}{1998} & 1999 & \multicolumn{1}{c}{2000} & 2001 \\
\hline I. PIB precios de mercado & 236504 & 257439 & 258031 & 272149 & 292858 & 298948 & 283523 & 284203 & 268696 \\
\hline II. Saldo comercial & -5686 & -7924 & -1100 & -1822 & -6571 & -7613 & -4950 & -1801 & 3543 \\
\hline III. Superávit primario total & 5645 & 2864 & 2710 & -657 & 1468 & 2587 & 3455 & 2864 & 1455 \\
\hline $\begin{array}{l}\text { IV. Superávit primario } \\
\text { sin privatización }\end{array}$ & 5121 & 2132 & 1538 & -1287 & 1163 & 2497 & 876 & 2720 & 1395 \\
\hline V. Intereses & 2914 & 3150 & 4084 & 4608 & 5745 & 6660 & 8224 & 9656 & 10175 \\
\hline V-II. Déficit de divisas & 8600 & 11074 & 5184 & 6430 & 12316 & 14273 & 13174 & 11457 & 6632 \\
\hline $\begin{array}{l}\text { V-IV. Déficit de recursos } \\
\text { fiscales }\end{array}$ & -2207 & 1019 & 2545 & 5889 & 4582 & 4170 & 7348 & 6936 & 8780 \\
\hline $\begin{array}{l}\text { V-III. Déficit de recursos } \\
\text { fiscales con privatización }\end{array}$ & -2731 & 286 & 1373 & 5264 & 4277 & 4074 & 4768 & 6792 & 8719 \\
\hline
\end{tabular}

Fuente: Elaboración propia con datos del Ministerio de Economía.

En las tres últimas filas, los déficit tienen signo positivo.

El signo negativo implica exceso de recursos o divisas sobre las necesidades del pago de intereses

La insuficiencia fiscal estaba mucho más relacionada con una estructura contradictoria en materia de recaudación, pues mientras se buscaba sostener la competitividad y las ganancias a costa de los salarios, la principal fuente de recaudación fueron los impuestos indirectos. La solución a la debilidad fiscal consistía, pues, en incrementar las contribuciones directas o estimular una distribución progresiva del ingreso. Pero ambos aspectos contradecían las características generales del modelo ${ }^{14}$. Por eso, no sorprende

13 Críticas a dicha interpretación pueden encontrarse en Damil, Frenkel y Juvenal, 2004; Gaggero, 2004.

14 Cfr. Musacchio, 2004. 
que las medidas aplicadas agravaran los problemas estructurales existentes. Una alternativa habría sido una mayor dinámica productiva y del empleo, pero los alicientes a la inversión financiera o a una racionalización en los procesos productivos dejaban poco margen para ello.

Otra característica del modelo procede de la necesidad insatisfecha de divisas por medio del comercio exterior. El modelo que inspiraba la marcha de la economía argentina fue el enfoque monetario de la balanza de pagos, inspirado en el modelo Mundell-Flemming. Una de los planteamientos de dicho enfoque es que una economía abierta a los flujos de capital permite un ajuste de las tasas de interés internas convergente con la tasa de interés internacional. Esto supone, empero, equilibrio en la cuenta corriente o una sensibilidad extrema de los flujos de capital a las diferencias entre ambas tasas de interés. La existencia de un desequilibrio permanente en la cuenta corriente obligó a atraer fondos desde el exterior en forma de deudas o inversiones directas. La sensibilidad de estos recursos fue bastante menor a lo esperado, por lo que el equilibrio en la balanza de pagos se logró a expensas de una tasa de interés muy elevada y el ofrecimiento de ganancias extraordinarias para las inversiones directas. Pero ambas formas traen como consecuencia un incremento de los egresos futuros, de modo que sólo sirven si son usadas de manera puntual. Como política de largo plazo, suponen un crecimiento incesante de la tasa de interés que, a la larga, se vuelve explosivo ${ }^{15}$.

De allí que la crisis estaba en marcha prácticamente desde el momento en que comenzó a funcionar la convertibilidad, y se agravó con las medidas adoptadas para reforzar la competitividad y las ganancias. En consecuencia, se generaron condiciones extremadamente precarias para gran parte de las actividades productivas y se gestó una burbuja especulativa sin ningún sustento en la economía real; por ello mismo, estaba destinada a explotar en cualquier momento.

Lo ocurrido con las brechas fiscal y externa puede ser descrito gráficamente como se muestra en la ilustración 2.

El desplazamiento de ambas curvas, representado por las líneas enteras S-C y S-F, a una posición como la resultante de las líneas punteadas $\mathrm{S}^{\prime}-\mathrm{C}^{\prime}$ y $\mathrm{S}^{\prime}-\mathrm{F}^{\prime}$, derivó en una situación en la que el punto de cruce de ambas brechas -informalmente, el punto de equilibrio del modelo- se produjo con niveles significativos de déficit comercial y fiscal.

En este esquema, la posibilidad de atender simultáneamente a las dos restricciones no era más que ilusoria: un proceso de crecimiento que oxigenara las cuentas fis-

15 Cfr. Musacchio, 2002. 


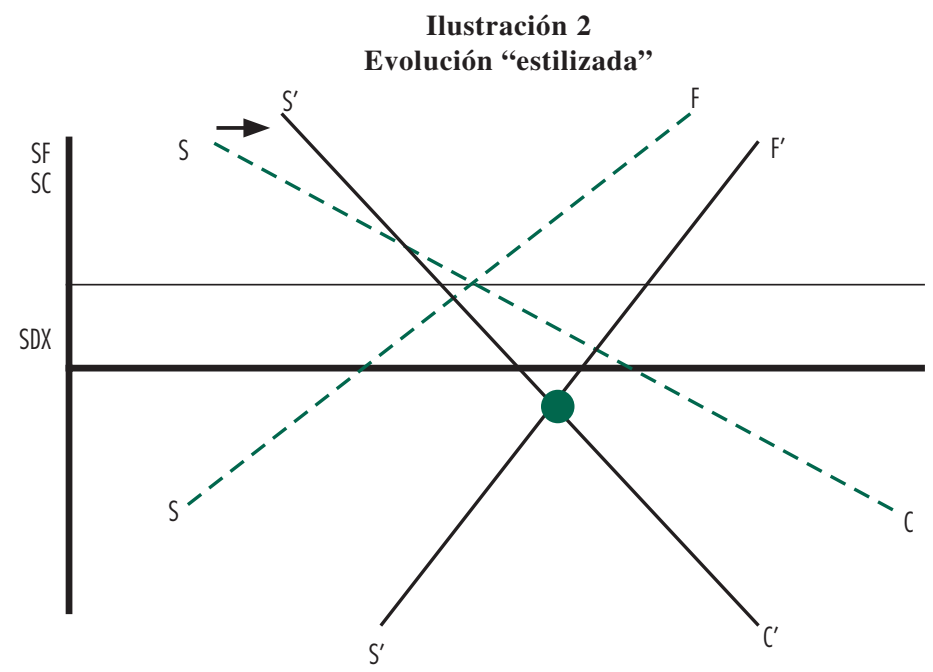

cales amplificaba el déficit comercial. Por el contrario, si se deseaba reequilibrar las cuentas externas, la recesión que acompañaba al proceso afectaba el balance fiscal. Si se siguen con atención las fases de la coyuntura entre 1991 y 2001, se podrá advertir con facilidad que los momentos de calma fiscal coincidieron con turbulencias externas y viceversa, lo cual, según nuestro planteamiento, no es mera coincidencia, sino el resultado previsible de la política económica aplicada, que conducía a un síndrome de la manta corta: al tapar los pies, se destapa el cuerpo, y al contrario.

Lo destacado, empero, es que los desequilibrios externos y fiscales tendieron a agravarse el uno al otro, tensión que se resolvía recurriendo al mercado financiero, pero a costa de aumentar la tasa de interés. Lejos de igualar la tasa de interés interna con la internacional, la liberalización financiera tendió a ampliar su diferencia, estimulando la especulación financiera e inhibiendo la actividad productiva. La crisis no fue, pues, consecuencia de un relajamiento del Plan de Convertibilidad o de cierta lentitud y falta de radicalidad en las reformas y en la liberalización de los mercados, sino, precisamente, un derivado del esquema de políticas propuesto. Por lo tanto, la perduración del plan durante una década no puede considerarse un mérito, sino una incomprensible ceguera técnica ante conflictos y tensiones macroeconómicas y productivas, así como políticas y sociales, cuyos efectos serán muy difíciles de erradicar. Indudablemente, como muestran muchos momentos de la historia argentina, tal ceguera técnica se enlaza con la aguda visión de grupos concentrados internos y extranjeros que se benefician de tales políticas y las sostienen tanto como les permitan obtener réditos. Luego, tienden a “socializar" las pérdidas, endosándoselas al Estado. 


\section{El golpe de timón de 2002}

La pregunta que surge inmediatamente es si las condiciones que se articularon a partir de 2002 son suficientes para evitar que una crisis como la de entonces no se repita o, más concretamente, si esta crisis revirtió la jerarquía de las formas institucionales tanto como para resolver la inconsistencia de fondo que conducía a los recurrentes desequilibrios externos e internos.

La crisis fue la muestra más clara de que el modelo resultaba insostenible. El experimento de la convertibilidad como forma de conciliar los desequilibrios recurrentes, lejos de ser efectivo, profundizó aún más las inconsistencias estructurales e institucionales. Esto puso al descubierto la existencia de una tercera brecha implícita, en la que el ahorro excedía al consumo y la inversión, deteriorando la base real de la economía por la apreciación del tipo de cambio y promoviendo una tendencia potencialmente contractiva, que generó las bases para la formación de una burbuja especulativa y para la fuga de capitales al exterior y disparó las tensiones sociales que confluyeron en el estallido de diciembre de 2001.

La violenta crisis obligó a ajustar las variables de manera forzosa y forzada. La depresión interna liberó saldos exportables y, especialmente, comprimió de manera drástica las importaciones. Se lograba así reforzar el superávit comercial que había aparecido con el inicio de la depresión. La reforma de los ingresos públicos y la inflación con congelamiento de gastos nominales cristalizaron en un superávit fiscal creciente. Por último, el default de parte de la deuda externa redujo la necesidad de financiamiento de su servicio. En estas condiciones, la política se tornó más coherente en el corto plazo y permitió volver a un sendero de crecimiento, aunque a costa de una situación social que, en los primeros meses de 2002, fue de las más delicadas de la historia argentina.

Un tema fundamental que alteró los parámetros de las brechas fiscal y externa de manera simultánea fue la introducción de retenciones a las exportaciones. Con ellas se reforzaron de manera ostensible las cuentas públicas. También, la introducción del nuevo tributo elevó la relación recaudación-producto y morigeró el impacto de la devaluación sobre los ingresos públicos al introducir un impuesto "dolarizado". Finalmente, empezó a tejer un lazo entre ambas brechas, pues cuando se incrementan las exportaciones, también lo hace la nueva porción de la recaudación.

Por otra parte, el cese de pagos parcial y la posterior reestructuración de la deuda, que postergó los pagos y convirtió en pesos una parte de los pasivos, redujeron las necesidades de saldos fiscales y comerciales para equilibrar las brechas. 
Gráficamente, el primer impacto esperado de las nuevas condiciones de la política económica asociadas a la devaluación y a la introducción de retenciones a las exportaciones puede interpretarse como un desplazamiento hacia la izquierda de la curva SF, mientras se mantenía fija la relación comercio exterior-ingreso.

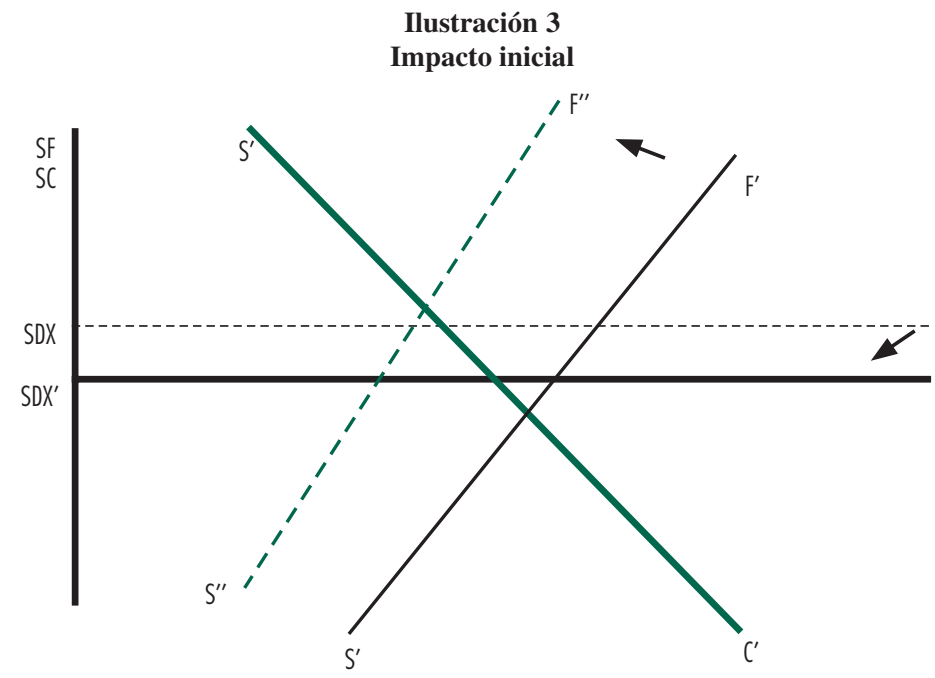

Poco a poco, la recuperación de los salarios y el crecimiento económico sostenido gestaron una precaria pero ostensible recuperación. Las nuevas condiciones reforzaron el panorama externo desde varios puntos de vista. En primer lugar, la devaluación permitió mejorar la posición competitiva de la producción local, lo que alentó las exportaciones y la sustitución de importaciones. A esto se le añadió un aumento de los precios externos que incrementó el valor de las ventas externas.

Desde una perspectiva de mediano plazo, el efecto macroeconómico de estas nuevas circunstancias (plasmado en la ilustración 4) puede ser descrito así: los efectos sobre el comercio exterior provocan un desplazamiento de la curva hacia afuera y hacia la derecha, vinculando un mismo nivel de ingreso con un saldo comercial más elevado. A su vez, la sustitución de importaciones trae como consecuencia que el impacto del crecimiento sobre la demanda de productos provenientes del exterior sea menor, es decir, reduce la elasticidad ingreso de las importaciones -las variaciones porcentuales de las compras externas ante cambios en el nivel de ingreso-, de modo que la pendiente de la curva $S " C "$ es menor que la de la curva $\mathrm{S}^{\prime} \mathrm{C}^{\prime}$. 
Ilustración 4

Impacto de mediano plazo
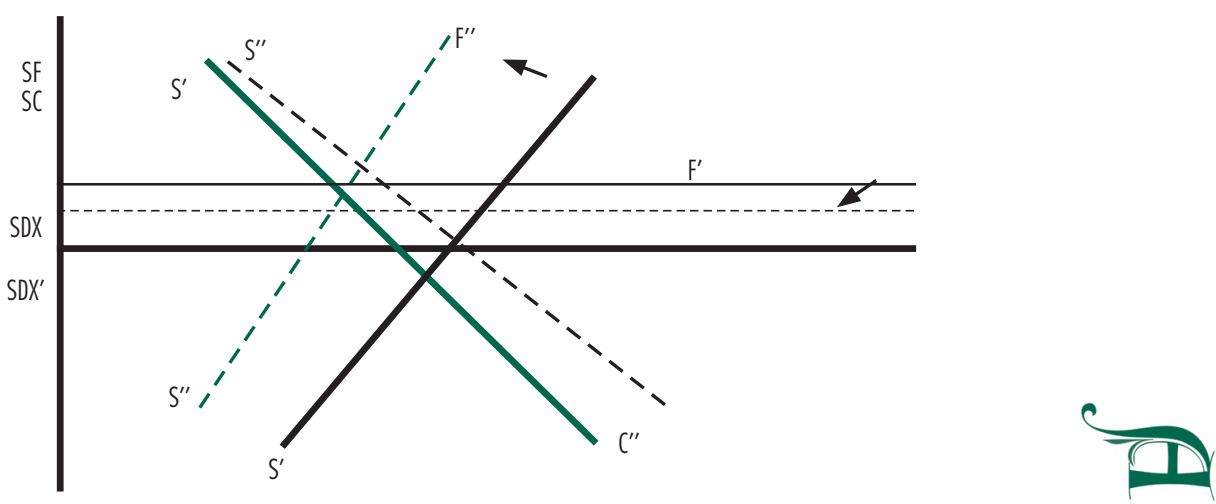

Si los movimientos esperados se plasmaran en la evolución real de las variables, podría afirmarse que, desde la devaluación, la evolución contradictoria de las brechas externa y fiscal debería haberse moderado notablemente, dando paso a una situación de excedentes simultáneos en el frente externo y fiscal. Esta situación fue, efectivamente, la que permitió retomar el servicio de la deuda externa con los acreedores privados luego de una áspera renegociación y sostener el servicio de la deuda con los organismos de crédito multilaterales, incluyendo la cancelación total anticipada de los pasivos con el FMI. Aun así, las reservas de divisas del Banco Central se incrementaron y el excedente fiscal permitió relajar el presupuesto que, inicialmente, había sido congelado de manera estricta. De esa forma, el gobierno se permitió ciertas "licencias", como la recomposición de los salarios docentes o el relanzamiento tímido de la obra pública, que incrementaron el gasto sin hacer mella en la recaudación, contradiciendo la prédica neoliberal que advertía el carácter inflacionario de tales medidas. En realidad, dado que el fisco registró sucesivos superávit, debería esperarse un efecto deflacionario y no inflacionario, incluso si el gasto crece, pues el Estado retira mas recursos de los que agrega y, por ende, retrae la demanda efectiva.

En el frente externo, la buena coyuntura permitió combinar un crecimiento de la producción con un aumento del superávit comercial, mientras el retorno de algunas inversiones directas o financieras desde el exterior oxigenó las cuentas restantes de la balanza de pagos. La tensión insoportable sobre el mercado de divisas cedió y condujo a una tensión a la baja del precio del dólar, sostenido sólo por la decisión del Banco Central de la República Argentina y del Banco Nación con compras permanentes en el mercado abierto. 
En el caso del sector externo, las implicaciones del análisis planteado en la ilustración 4 puede advertirse con singular claridad en la gráfica 3, donde se comprueba el desplazamiento de la línea de tendencia hacia arriba y hacia afuera, señalando un saldo comercial superior a igual nivel de PIB, así como una menor pendiente, es decir, una menor sensibilidad del saldo ante variaciones del producto.

Gráfica 3

Relación entre el saldo comercial y el PIB, periodos 1998-2001 y 2002-2007 (millones de pesos corrientes)

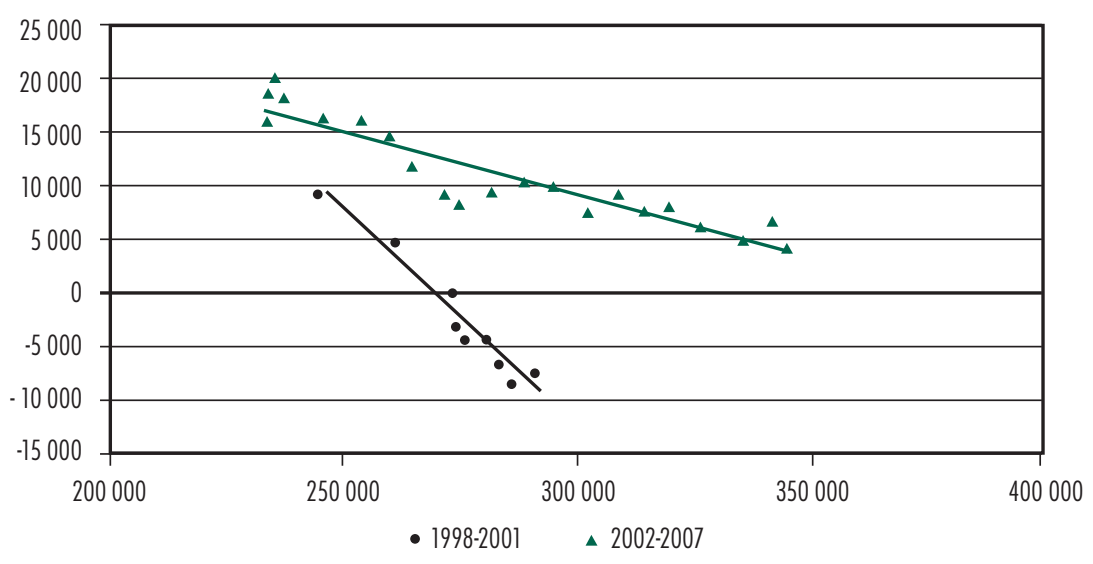

Fuente: Elaboración propia con base en datos del Ministerio de Economía.

La comparación se ve sesgada por los cambios en la estructura de precios relativos introducida por la devaluación y las alteraciones en los precios internacionales. Pero precisamente la devaluación proponía tal cambio de la estructura de precios como elemento central de la nueva política económica, de modo que no es un efecto exógeno. Por eso, también juzgamos más relevante, en este contexto, la comparación en precios corrientes como la expresada en la gráfica 3. En cambio, los aumentos de los precios internacionales actúan como un shock externo positivo y deberían tomarse como transitorios, especialmente ahora que la tendencia parece haber comenzado a revertirse.

En el caso de la brecha fiscal, aunque el efecto no fue tan marcado ni tan inmediato como en el caso de la brecha externa, también las evidencias permiten sostener la hipótesis que planteamos, como puede verse en la gráfica 4 .

Se advierte una curva menos sensible y mucho más volátil para el periodo 20022006 que para el cuatrienio previo. Esto denota, además, que más allá de algunas modificaciones, una reforma estructural profunda de las cuentas públicas queda pendiente, a excepción de la introducción de retenciones a las exportaciones. Por lo tanto, la recuperación fiscal se debió sobre todo al intenso crecimiento del PIB. 
Gráfica 4

Relación entre el saldo fiscal y el PIB, periodos 1998-2001 y 2002-2006

(millones de pesos corrientes)

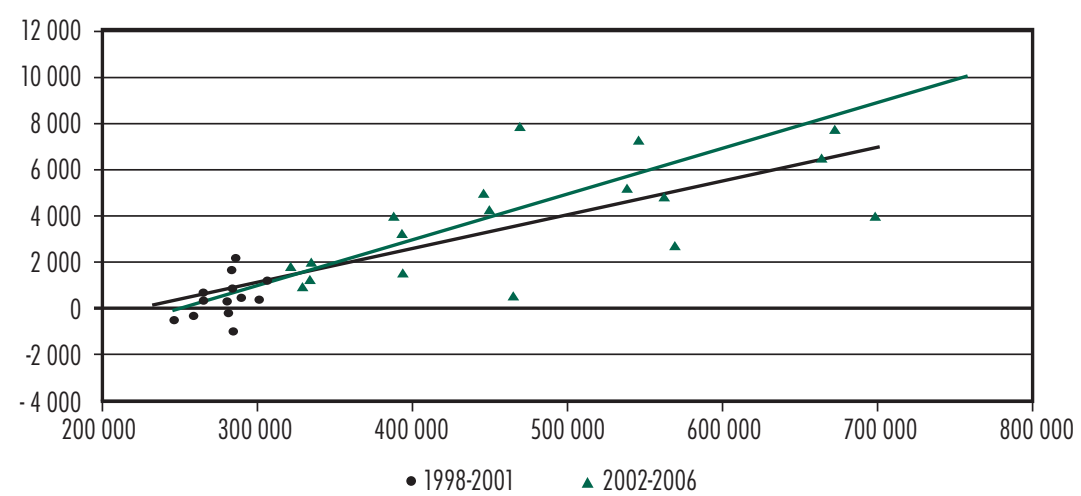

Fuente: Elaboración propia con base en datos del Ministerio de Economía.

La evolución de las variables macroeconómicas luego de la devaluación y las nuevas medidas tributarias, la reestructuración de la deuda y la nueva matriz externa permiten afirmar que en el primer lustro posterior a la crisis, las brechas externa y fiscal han logrado una trayectoria más armónica, compatible con los servicios de la deuda. Esto redujo la necesidad de atraer recursos externos con aumentos de las tasas de interés y la reevaluación del tipo de cambio real; de allí que no se recrearan las bases para la aparición de burbujas financiero-especulativas tan proclives a estallar en crisis incontenibles. Sin embargo, el interrogante es si la recuperación y el alcance de los superávit externo y fiscal compatibles con las necesidades del servicio de la deuda pueden mantenerse en el futuro, mientras se sostiene el proceso de crecimiento económico y se atiende a las necesidades de mayor inversión y de una distribución más equitativa del ingreso. En síntesis, la pregunta es si desde una perspectiva técnica los cambios recientes bastan para afirmar que se encuentra en marcha un modelo de desarrollo sustentable con un horizonte despejado en el largo plazo.

La devaluación y una política económica más activa contribuyeron a asentar algunas bases más sólidas que las de la década de 1990 y cerraron muchos de los desequilibrios macroeconómicos. También es cierto que el nuevo esquema surgió en el contexto de una coyuntura internacional especialmente favorable, que permitió la fuerte expansión de las exportaciones a partir de mayores volúmenes y, sobre todo, precios más altos, con una mejora de más de $30 \%$ en los términos del intercambio entre 2003 y 2007. 
Sobre esa coyuntura, fue posible aplicar nuevos tributos como las retenciones, que derramaron parte de los beneficios de las condiciones externas extraordinarias sobre las cuentas públicas, evitando además una mayor violencia de los efectos negativos de la devaluación sobre la distribución del ingreso.

Cuadro 2

Principales variables observadas. En pesos corrientes, salvo * en dólares

\begin{tabular}{|c|c|c|c|c|c|c|}
\hline & 2002 & 2003 & 2004 & 2005 & 2006 & 2007 \\
\hline I. PIB Precios de mercado & 312580.00 & 375909.00 & 447643.00 & 537939.00 & 65439.00 & 812456.00 \\
\hline II. Saldo comercial ${ }^{\star}$ & 17178.00 & 16805.00 & 13265.00 & 13087.00 & 13958.00 & 13255.00 \\
\hline III. Superávit primario total & 2260.20 & 8688.10 & 17360.80 & 19661.20 & 23164.80 & 25718.60 \\
\hline IV. Superávit primario sin privatización & 2255.70 & 8676.90 & 17332.70 & 19622.60 & 23157.90 & 25670.10 \\
\hline V. Intereses & 6809.50 & 6882.80 & 5703.00 & 10243.10 & 11541.80 & 16422.50 \\
\hline En moneda local & 981.40 & 1882.90 & 1872.80 & 3123.40 & 3744.40 & 5873.10 \\
\hline En moneda extranjera & 5828.10 & 4999.90 & 3830.20 & 7119.70 & 7797.40 & 10609.40 \\
\hline Tipo de cambio & 3090.40 & 2949.10 & 2941.50 & 2923.30 & 3074.00 & 3715.40 \\
\hline VI. Intereses en moneda extranjera medidos en dólares & 1885.89 & 1695.38 & 1302.14 & 2435.49 & 2536.57 & 3405.50 \\
\hline VI. Déficit de divisas* & 15291.67 & 15109.22 & 11962.46 & 10651.17 & 11421.73 & 9849.60 \\
\hline VIII. Déficit de recursos fiscales & 4553.80 & -1794.10 & -11629.70 & -9379.50 & .11616 .10 & -9247.58 \\
\hline IX. Déficit de recursos fiscales con privatización & 4549.30 & -7805.30 & -11657.80 & -9418.10 & -11623.00 & -9296.08 \\
\hline
\end{tabular}

Fuente: Elaboración propia con base en datos del Ministerio de Economía.

En las tres últimas filas, los déficit son los que tienen signo positivo.

Signo negativo implica exceso de recursos o divisas sobre las necesidades del pago de intereses

Sin embargo, el sector externo refleja un cambio estructural débil, por cuanto la composición de las exportaciones no sufrió alteraciones significativas, y sirve como ejemplo de la precariedad de algunas de las mejoras ${ }^{16}$. En ese sentido, es posible afirmar que muchos de los conflictos preexistentes solamente fueron desplazados del centro de la escena, mas no resueltos desde la raíz. Así, la ligera sustitución de importaciones no llega a constituir aún un verdadero modelo de desarrollo económico y social, pues no busca -ni se ha plasmado en- un cambio estructural apoyado por políticas activas. El gran avance de las exportaciones fue resultado de condiciones excepcionales de precios disparados por la presencia de una demanda china que no cesa de crecer. Pero la concentración de las ventas externas en unos cuantos productos, que se ha mantenido inamovible, también permite vislumbrar la fragilidad del superávit comercial, que poco a poco, incluso antes del estallido de la crisis, ha sufrido algunos

16 Cfr. Musacchio, 2004b; Musacchio y Robert, 2006. 
impactos de advertencia. Por ejemplo, el gran superávit con China, que se consideraba uno de los datos sobresalientes de los años venideros, se ha revertido a medida que aquel país comenzó a colocar productos en Argentina, especialmente luego de un convenio sobre el que oportunamente habíamos expuesto nuestras dudas ${ }^{17}$.

De la misma manera, la distribución del ingreso es otro problema postergado, con algunas soluciones parciales para los sectores más necesitados, pero sin nuevas fórmulas de regulación que contemplen pautas estables y generales. Tampoco se produjo una reforma tributaria que redujera los impuestos indirectos y cargara aún más la recaudación sobre contribuciones directas, como parte de una nueva política de ingresos. La crisis energética que padece el país es la punta del iceberg de un problema más profundo: la falta de inversión pública en infraestructura básica. El problema no es atribuible a las políticas recientes, sino que se arrastra por más de tres décadas y mejoró ligeramente hace poco tiempo. Pero el esfuerzo necesario para superar 30 años de deterioro es una carga que las cuentas fiscales, en las condiciones actuales, no podrían sostener cateris paribus. La manta sigue siendo aún muy corta tanto por la estructura regresiva de la recaudación como por la insuficiente magnitud de la riqueza creada.

Por otra parte, el cronograma de pagos de la deuda externa para la próxima década indica que los superávit externo y fiscal deberán, como mínimo, mantenerse. Y ni siquiera así alcanzan a cubrir el servicio de la deuda, por lo que obligan al gobierno a recurrir moderada pero efectivamente al crédito para completar las transferencias. Por lo tanto, el problema de la deuda permanece latente.

La tregua que ofreció la coyuntura posterior a 2003 permitió un panorama más favorable para el quiebre del modelo neoliberal, sobre el cual comenzar a pensar en un nuevo proyecto de desarrollo económico y social de largo plazo. Sin embargo, algunos de los problemas fundamentales que afectan a la estructura económica del país persisten y generan el interrogante sobre lo que ocurrirá con el derrame de la crisis internacional. En el fondo, se trata de desentrañar qué cambió y qué se mantuvo de las viejas estructuras económicas, sociales y productivas.

17 Cfr. Musacchio, Fiszbein y Braude, 2004. 


\section{Los primeros avatares de la crisis}

La crisis internacional supone una drástica transformación de la coyuntura sobre la que se asienta el programa macroeconómico argentino. A diferencia de crisis internacionales anteriores, la inexistencia de una burbuja financiera especulativa y la independencia de un endeudamiento externo creciente para sostener la política económica ha evitado las conocidas explosiones que Argentina sufría cada siete u ocho años. El quiebre de las finanzas internacionales y la retracción de los flujos de capitales de corto plazo no parecieron alterar drásticamente la coyuntura externa. Las reservas acumuladas en los años previos permitieron hacer frente a una salida de capitales que comenzó a insinuarse en el tercer trimestre de 2007 y se profundizó a lo largo de 2008, acumulando en ese periodo casi 12000 millones de dólares.

La nueva situación pone de manifiesto la solidez macroeconómica al inicio de la crisis, pero llama la atención sobre los futuros acervos de divisas y genera una creciente presión sobre el tipo de cambio, que luego de estar sometido durante largo tiempo a una presión bajista, muestra hoy la tendencia -estructural para Argentinahacia el alza del precio de las divisas. El alto acervo de reservas, junto con la política de sostener el tipo de cambio en momentos de presión a la baja, han permitido hasta el momento una devaluación "ordenada", muy suave y paulatina, estilo crawling peg, que contrasta con las maxidevaluaciones habituales de crisis anteriores y con las fluctuaciones violentas que se vieron en el mundo desde el inicio de la debacle.

La política económica actual tiene su flanco más vulnerable en la balanza comercial, pues es allí donde se encuentra la clave para evitar turbulencias en el sector externo y también una de las fuentes vitales de la recaudación. En ese sentido, el inicio de 2009 presenta un cuadro contrastante. Si bien el saldo comercial se encuentra estabilizado, el comercio exterior ha caído sostenidamente desde el último trimestre de 2008, y los datos comparativos del primer bimestre de 2009 frente al mismo periodo del año anterior son preocupantes. Las exportaciones se contrajeron $24 \%$ con respecto al mismo mes de 2007, mientras las importaciones se redujeron 37 por ciento.

Contrariamente a lo que ocurrió en la fase de auge, la reducción del comercio exterior en el primer bimestre de 2009 no tuvo como causa principal una disminución de los precios, sino de las cantidades; los términos del intercambio para el país continúan en un nivel mejor que a principios de 2007. Lo que ocurre es que se están desacelerando algunos sectores clave del comercio exterior argentino, como el automotriz o el siderúrgico, con la caída de la demanda externa y los problemas de algunos grupos transnacionales radicados en dichos sectores. Ello no implica, empe- 
ro, que no exista la posibilidad de un paulatino empeoramiento de los términos del intercambio, cuadro más acorde con las tendencias históricas y que pondría en tela de juicio la euforia de precios reciente en los commodities agrícolas o de las materias primas de origen mineral.

Ésa es la puerta de entrada de la crisis a la Argentina y, por lo tanto, inicialmente habría de afectar a los complejos exportadores industriales -sector automotriz, siderúrgico, químico- con una baja en la actividad, mientras que en el resto del sector exportador más bien debería percibirse una disminución de precios. Frente a las nuevas condiciones, el gobierno ha reaccionado estimulando la demanda interna con programas específicos para la compra de automotores o electrodomésticos, mientras procura defender el saldo comercial con sutiles restricciones a las importaciones.

Por su parte, el principal golpe en las importaciones se refleja en las compras de bienes de capital e insumos intermedios, que muestran un descenso de la tasa de inversión y, por lo tanto, de las bases del crecimiento de largo plazo.

El elemento central para percibir si Argentina se dirige hacia un ajuste caótico o hacia una desaceleración del crecimiento puede entenderse, una vez más, con el modelo de doble brecha. Al parecer, lo que sucede, y que se agravaría con una caída de precios, es que los niveles de recaudación y de superávit comercial serían más reducidos, a igual nivel de producto, de la manera en que se ve en la ilustración 5. El nivel de ese desplazamiento, cateris paribus, dependerá de la reducción de los precios, de la evolución de la demanda externa y de la caída consecuente de las retenciones.

El desplazamiento de la relación saldo fiscal-producto es consecuencia directa del impacto en la recaudación del descenso de las exportaciones, que disminuye las retenciones a las exportaciones. Los planes de asistencia a diferentes sectores, por medio de subsidios o desgravaciones impositivas, tienen un impacto similar. En esta fase inicial, los impactos negativos han sido compensados con la reestatización del sistema de jubilaciones y pensiones, que vuelve a poner en manos del Estado los recursos absorbidos en los últimos años por las administradoras privadas.

En el caso del sector externo, los impactos cruzados de reducción de precios y demanda externa, por un lado, y la desaceleración del crecimiento, la caída de las compras de bienes de inversión e insumos, por el otro, generan en el corto plazo un escenario incierto, pero también complejo a largo plazo. Por lo pronto, tanto en el frente fiscal como en el comercial, los saldos se mantienen superavitarios y esa tendencia aún no parece estar en riesgo. Sin embargo, la consistencia macroeconómica de mediano plazo todavía depende de que tales superávit sean suficientes para absorber el servicio de la deuda externa. Por lo tanto, tan importante como la evolución de precios, la demanda 


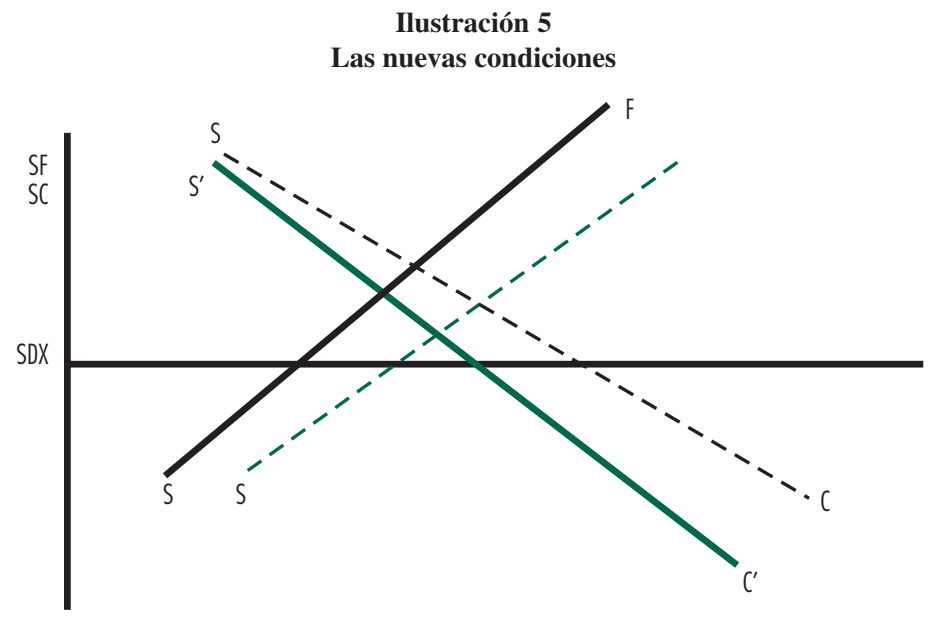

externa, la caída de las exportaciones agrícolas o del nivel de actividad interna resulta el cronograma de vencimientos de la deuda.

La coyuntura, hasta el momento, no ha puesto en jaque la capacidad de pago, pues los saldos permitirían cubrir las necesidades de financiamiento y, en dado caso, se podría recurrir prudentemente a la utilización de las reservas. El cronograma inmediato supone vencimientos que, en el nivel de PIB alcanzado, no parecen intolerables si se conjuga una política de pagos parciales, refinanciación de algunos créditos y la toma de alguna línea de créditos especial. En ese marco, las reservas pueden conjurar algún apuro. Sin embargo, algunas señales del propio gobierno -como la posibilidad de recurrir nuevamente a algún crédito del FMI o el acuerdo de compensación firmado recientemente con China-indican que las expectativas tampoco son del todo alentadoras.

Luego de siete años de abandono del modelo neoliberal, la crisis toma a Argentina en una situación contradictoria. Por un lado, la menor dependencia de flujos externos permite cubrir la etapa inicial de la crisis de una manera mucho más compacta y tranquila que en crisis anteriores. Pero, por otra parte, la inexistencia de un programa de desarrollo y cambio estructural de largo plazo ha impedido transformar por completo los parámetros de la economía y la sociedad. La extrema concentración sectorial de las exportaciones con un sesgo marcado hacia la explotación de recursos naturales, y la ausencia de una verdadera política de sustitución de importaciones fundada en una orientación explícita hacia sectores prioritarios mantienen una estructura extremadamente vulnerable, aunque dicha vulnerabilidad sea de naturaleza muy distinta a la de la década de 1990. 
Cuadro 3

Perfil de vencimientos de la deuda externa pública, 3er trimestre de 2008

\begin{tabular}{|l|r|r|r|r|r|r|r|r|}
\hline & \multicolumn{5}{|c|}{$\begin{array}{c}\text { Un año o menos } \\
\text { (meses) }\end{array}$} & \multicolumn{2}{|c|}{$\begin{array}{c}\text { Uno a dos años } \\
\text { (meses) }\end{array}$} & $\begin{array}{c}\text { Más de dos } \\
\text { años }\end{array}$ \\
\hline & $\begin{array}{l}\text { Presente } \\
\text { trimestre }\end{array}$ & $0-3$ & $4-6$ & $7-9$ & $10-12$ & $13-18$ & $19-24$ & \\
\hline Gobierno nacional & 6318.30 & 1439.50 & 894.50 & 1257.40 & 2095.80 & 2137.70 & 3290.00 & 81235.80 \\
\hline Principal & 6318.30 & 824.10 & 507.50 & 628.90 & 1621.30 & 1078.70 & 2255.00 & 49680.20 \\
\hline Intereses & & 615.40 & 387.00 & 628.50 & 474.50 & 1059.00 & 1035.00 & 37555.60 \\
\hline Autoridades monetarias & 0.0 & 3796.80 & 57.10 & 41.10 & 36.00 & 295.30 & 26.60 & 74.00 \\
\hline Capital & & 3762.10 & 20.60 & 12.50 & 0.00 & 245.60 & 17.60 & 63.90 \\
\hline Intereses & & 34.70 & 36.50 & 28.60 & 36.00 & 49.70 & 9.00 & 10.10 \\
\hline Servicio total & 6318.30 & 5236.30 & 951.60 & 1298.50 & 2131.80 & 2433.00 & 3376.60 & 81309.80 \\
\hline Capital & 6318.30 & 4586.20 & 528.10 & 641.40 & 1621.30 & 1324.30 & 2272.60 & 49744.10 \\
\hline Intereses & 0.0 & 650.10 & 423.50 & 657.10 & 510.50 & 1108.70 & 1044.00 & 31565.70 \\
\hline
\end{tabular}

Fuente: INDEC.

Por eso, la crisis también se manifiesta de una manera muy diferente. No ocasiona una ruptura explosiva como ocurrió en 1982, 1989 o 2001, sino una paulatina desaceleración del crecimiento. Las nuevas condiciones harán más difícil el impulso de un cambio estructural radical, dado que la capacidad de acumulación, de retención de los excedentes y de captación de recursos por parte del Estado para realizar las grandes obras de infraestructura que se requieren, se verá resentida. También hará más perceptibles los déficit en la estructura productiva y la necesidad de reforzar el mercado interno ante la debilidad de los impulsos provenientes del exterior. Por lo tanto, en peores condiciones "técnicas", un replanteamiento profundo del rumbo -pero no un retorno a los noventa- se hará imprescindible. 
Artana, Daniel, Ricardo López Murphy y Fernando Navajas, "La crisis económica argentina", en Daniel Artana y James Dorn (comps.), Crisis financieras internacionales-¿Qué rol le corresponde al gobierno?, Buenos Aires, FIEL, 2004.

Basualdo, Eduardo, "Las reformas estructurales y el Plan de Convertibilidad durante la década de los noventa. El auge y la crisis de la valorización financiera", Realidad económica, núm. 200, Buenos Aires, Instituto Argentino para el Desarrollo Económico, 2003.

, Sistema político y modelo de acumulación en la Argentina. Notas sobre el transformismo argentino durante la valorización financiera1976-2001, Buenos Aires, Universidad Nacional de QuilmesFLACSO-IDEP, 2002.

Becker, Joachim, "La reciente crisis financiera en Argentina, Brasil y Uruguay. Análisis comparativo", Documento de discusión global D3E, Montevideo, 2003.

Boyer, Robert y Julio Neffa (coords.), La economía argentina y su crisis (1976-2001). Visiones institucionalistas y regulacionistas, Buenos Aires, Miño y Dávila editoresCEIL-PIETTE CONICET-CDC, 2004.

Calvo, Guillermo y A. Izquierdo, Sudden Stops, the Real Exchange Rate and Fiscal Sustainability: Argentina's Lessons, Washington D.C., BID, 2002.

Damil, Mario, Roberto Frenkel y Luciana Juvenal, "Las cuentas públicas y la crisis de la convertibilidad en Argentina", en R. Boyer y J. Neffa (coords.), La economía argentina y su crisis (1976-2001). Visiones institucionalistas y regulacionistas, Buenos Aires, Miño y Dávila editores-CEIL-PIETTE CONICET-CDC, 2004.

Gaggero, Jorge, "La cuestión fiscal, huella de la historia política y económica", en R. Boyer y J. Neffa (coords.), La economía argentina y su crisis (1976-2001). Visiones institucionalistas y regulacionistas, Buenos Aires, Miño y Dávila editores-CEIL-PIETTE CONICET-CDC, 2004.

INDEC, www.mecon.gov.ar

Minsburg, Naum, Los guardianes del dinero: las políticas del FMI en la Argentina, Buenos Aires, Norma, 2003.
Musacchio, Andrés, "Ideología, tributación y crisis fiscal: Sísifo también es argentino", en Mario Rapoport (ed.), Crónicas de la Argentina sobreviviente. El presente en el espejo de la historia, Buenos Aires, Norma, 2004a.

" "Debilidades de la inserción comercial de Argentina, 1976-2003", en R. Boyer y J. Neffa, La economía Argentina y su crisis (1976-2003). Visiones institucionalistas y regulacionistas, Buenos Aires, Miño y Dávila editores-CEIL-PIETTE CONICETCDC, 2004b.

"La estrategia exportadora en Argentina: ¿necesidad, objetivo o utopía?”, en Julio Gambina (comp.), La globalización económico-financiera: su impacto en América Latina, Buenos Aires, CLACSO, 2002.

Musacchio, Andrés, Martín Fiszbein y Hernán Braude, "La expansión comercial con Asia. ¿Una estrategia novedosa o parche para el viejo modelo?", Informe económico IMA, núm. XXVI, Buenos Aires, noviembre-diciembre de 2004.

Musacchio, Andrés y Verónica Robert, “Opciones de inserción internacional y desarrollo económico y social en la Argentina del siglo XXI: Rupturas y continuidades después de la devaluación", en Julio Neffa y Héctor Cordone (comps.), Escenarios de salida de la crisis y estrategias alternativas de desarrollo para la Argentina, Buenos Aires, CEIL-PIETTE CONICET, 2006.

Mussa, Michael, Argentina y el FMI. Del triunfo a la tragedia, Buenos Aires, Planeta, 2002.

Plan Fénix, "Propuestas para el desarrollo con equidad", Enoikos. Revista de la Facultad de Ciencias Económicas, año 10, núm. 20, 2003.

, Hacia el Plan Fénix. Una alternativa económica, Buenos Aires, Prometeo, 2002.

Rapoport, Mario et al., Historia económica, política y social de la Argentina, $2^{\mathrm{a}}$ ed., Buenos Aires, Ariel, 2006.

Rodríguez, Carlos, Argentina en transición. La recesión 1998-2000, Buenos Aires, Temas GE, 2000.

Vitelli, Guillermo, "Las rupturas de la convertibilidad y el plan real: la reiteración de una misma historia", en Ciclos en la historia, la economía y la sociedad núm. 23, Buenos Aires, Fundación de Investigaciones Históricas, Económicas y Sociales-UBA-IIHES, 2002.

Vol. 40, núm. 159, octubre-diciembre / 2009 